Results The patient remained hemodynamically stable and conscious. She was discharged from the hospital with no record of complications.

Conclusions The number of high-risk frail patients is increasing. CSA allows the anesthetist to better manage these patients due to the fractionation of doses and greater control over the hemodynamic repercussions.

\section{Chronic pain management}

\section{REPETITIVE SHPENOPALATINE GANGLION BLOCK USING THE TX360 NASAL APPLICATOR FOR THE TREATMENT OF TRIGEMINAL NEURALGIA: A PILOT STUDY}

${ }^{1} \mathrm{~A}$ Tsaroucha*, ${ }^{1} \mathrm{C}$ Orfanou, ${ }^{2} \mathrm{~N}$ Fyrfiris, ${ }^{1} \mathrm{~A}$ Melemeni, ${ }^{1} \mathrm{I}$ Siafaka, ${ }^{1} \mathrm{~K}$ Theodoraki. ${ }^{1}$ Aretaieio Hospital, National and Kapodistrian University of Athens, Athens, Greece; ' ${ }^{2} G e n e r a l$ Oncology - Anticancer Hospital of Athens 'Agios Savvas', Department of Anesthesiology, Athens, Greece

\subsection{6/rapm-2021-ESRA.71}

Background and Aims Sphenopalatine ganglion (SPG) is situated in the posterior nasal cavity and belongs to the cranial part of the autonomic nervous system. Trigeminal neuralgia (TGN) is currently considered as an indication for SPG block, especially in medication-resistant cases. The aim of this study was to assess the effectiveness of the SPG block for the treatment of trigeminal neuralgia, using a noninvasive transnasal approach, by delivering local anesthetic with the alternative device Tx360 nasal applicator.

Methods Four patients (three women, aged 67, 56 and 75 and a man, aged 58) presented with either classical or atypical, V2 (maxillary branch) or V3 (mandibular branch) TGN, partly or completely drug-resistant. Pain severity during paroxysmal attacks was recorded using the visual analogue scale and it scored between 8 and 9 for all patients. The SPG block was achieved using the Tx360 nasal applicator in order to deliver $0.3 \mathrm{ml}$ of xylocaine $2 \%$, once a week, for 6 to 8 weeks. Participants in this study received up to 8 SPG blocks.

Results All patients reported significant pain relief and were completely symptom-free after the $2^{\text {nd }}$ or $3^{\text {rd }}$ application. This favorable outcome lasted for up to 3 months for each case. No significant adverse events were noted.

Conclusions Data extracted from this pilot study suggest that repetitive SPG block with the $\mathrm{Tx} 360$ nasal applicator may constitute an easy, rapid, safe and efficient treatment of trigeminal neuralgia. Further relative double-blind, randomized studies are required in order to draw solid conclusions.

\section{IMPROVEMENT OF HEALTH-RELATED QUALITY OF LIFE AND FUNCTIONAL DISABILITY IN PATIENTS WITH FBSS PAIN AND IMPLANTED WITH A RECHARGEABLE SPINAL CORD STIMULATION SYSTEM}

${ }^{1} \mathrm{R}$ Buschman* ${ }^{2} \mathrm{~A}$ Calodney, ${ }^{3} \mathrm{~J}$ Hatheway, ${ }^{4} \mathrm{~S}$ Eldabe ${ }^{5} \mathrm{~L}$ Cantin, ${ }^{6} \mathrm{E}$ Buchser, ${ }^{7} \mathrm{~N}$ Grunow, ${ }^{8} \mathrm{R}$ Slangen. ${ }^{1}$ Medtronic Trading NL B.V., Eindhoven, Netherlands; ${ }^{2}$ Precision Spine and Care, Tyler, USA; ${ }^{3}$ Northwest Pain Care, Spokane, USA; ${ }^{4}$ The James Cook University Hospital, Middlesbrough, UK; ${ }^{5}$ Hôpital Enfant-Jésus, Québec, Canada; ${ }^{6}$ Hôpital de Morges, Morges, Switzerland; ${ }^{7}$ Clinical Biostatistics, Medtronic, plc, Minneapolis, USA; ${ }^{8}$ Post Approval Clinical Surveillance, Medtronic, Maastricht, Netherlands
Background and Aims The Product Surveillance Registry (PSR, Medtronic) is a prospective, long-term, multicentre registry to monitor the performance and safety of Medtronic Spinal Cord Stimulation (SCS) systems. We present an exploration of the effect of SCS with 2 rechargeable systems (RestoreSensor and Intellis) in patients with Back Pain of Failed Back Surgery Syndrome (BP-FBSS) on Health-Related Quality of Life (HRQoL), and functional disability (Oswestry Disability Index, ODI).

Methods Patients with a diagnosis of BP-FBSS, a general pain score $\geq 5$, and an initial implant were included. EQ-5D and ODI scores were summarized at baseline, 6-months, and for the change from baseline to 6-months (paired t-tests evaluated within-group change).

Results EQ-5D UK scores showed statistically significant and clinically relevant improvements for both RestoreSensor and Intellis from baseline to 6 months; $0.47 \pm 0.21$ to $0.57 \pm 0.28$ $(\mathrm{n}=78 ; \mathrm{P}=0.001)$, and $0.45 \pm 0.25$ to $0.60 \pm 0.26 \quad(\mathrm{n}=82$; $\mathrm{P}<0.0001)$, respectively.

ODI scores reduced from $51.1 \pm 13.3$ to $45.1 \pm 16.4(n=76$; $\mathrm{P}=0.001)$ and $50.9 \pm 12.6$ to $39.3 \pm 16.6(\mathrm{n}=85 ; \mathrm{P}<0.0001)$, for RestoreSensor and Intellis, respectively.

Conclusions To assess clinical relevance, research suggest a minimum clinically important difference (MCID) of 0.081 in EQ-5D in a subgroup of patients with back pain and 9.2 in ODI in patients with FBSS. Our analysis shows statistically significant and clinically relevant improvements in health-related quality of life, and functional disability from baseline to 6months in patients diagnosed with BP-FBSS and implanted with a rechargeable SCS-system.

\section{THE ROLE OF ULTRASOUND GUIDED PULSED RADIOFREQUENCY (PRF) FOR THE TREATMENT OF RHIZARTHROSIS: A CASE REPORT}

${ }^{1} \mathrm{P}$ Fusco, ${ }^{2} \mathrm{~W}$ Ciaschi, ${ }^{3} \mathrm{~F}$ Angelucci ${ }^{*},{ }^{3} \mathrm{M}$ Di Carlo, ${ }^{3} \mathrm{~S}$ Tullj, ${ }^{3} \mathrm{M}$ Celniku, ${ }^{3} \mathrm{~F}$ Marinangeli. '1Department of Anesthesia and Intensive Care, San Salvatore Academic Hospital, L'Aquila, Italy; '2Department of Anesthesia and Intensive Care, F. Spaziani Academic Hospital, Frosinone, Italy; ${ }^{3}$ University of L'Aquila, Department of Life, Health and Environmental Sciences, L'Aquila, Italy

\subsection{6/rapm-2021-ESRA.73}

Background and Aims Rhizarthrosis is a disabling condition of the hand that affects $30 \%$ of postmenopausal women ${ }^{1}$. It commonly treated with physiokinesitherapy, non-steroidal antiinflammatory drugs, intra-articular corticosteroid injections ${ }^{2}$ and trapeziectomy.

Intra-articular PRF produces an electric field that disrupts smaller pain-carrying fibers of the synovial lining and reduces the levels of cytokines in the joint microenvironment ${ }^{3}$.

Methods This report describes one case in which the ultrasound guided pulsed radiofrequency of the radial nerve offered a safe treatment for the management of chronic rhizarthrosis pain. The case was a 74-years-old man with a 12 month history of severe pain at the thumb basal joint. At his first visit, the VAS measured 8/10. The patient was sitting on the operating table with the trapezium metacarpal joint up After preparation of the radial region, the sterile linear high frequency probe was longitudinally placed on the joint

(figure 1). Then, a 22 G $50 \mathrm{~mm}$ radiofrequency needle was introduced near the superficial radial. nerve (figure 2). Subsequently a thermocouple connected to a radiofrequency generator was inserted to reproduce the patient's paresthesia 